\title{
Glucose adulteration in Saudi honey with visible and near infrared
}

spectroscopy

\section{Abdul M. Mouazen; Noura Al-Walaan}

Environmental Science and Technology Department, Cranfield University, Cranfield, Bedfordshire, MK43 OAL, United Kingdom

E-mail of corresponding author: a.mouazen@cranfield.ac.uk

\section{ABSTRACT}

This paper reports on the implementation of visible and near infrared spectroscopy (visNIRS) for the detection of glucose concentration in a mixture of Saudi and imported honey samples adulterated by glucose syrup of five concentrations of $0,5,12,19$ and $33 \mathrm{~g} / 100 \mathrm{~g}$. Honey samples were scanned in trans-reflectance mode with an AgroSpec mobile, fibre type, vis-NIR spectrophotometer (tec5 Technology for Spectroscopy, Germany), with a measurement range of $305-2200 \mathrm{~nm}$. The entire data set of 345 spectra was randomly divided into calibration (70 \%) and prediction (30\%) sets. The first group was subjected to a partial least squares (PLS) regression analysis with a leave-one-out cross-validation to establish a calibration model for the prediction of glucose concentration, whereas the second group was used to validate the PLS model.

For the cross-validation the values for root mean square error of prediction (RMSEP), coefficient of determination $\left(R^{2}\right)$ and ratio of prediction deviation (RPD), which is the standard deviation divided by RMSEP were $4.52 \mathrm{~g} / 100 \mathrm{~g}, 0.85$, and 2.53 , respectively. A slightly lower range of accuracy was obtained in the prediction set, with RMSEP, $R^{2}$ and RPD values of $5.56 \mathrm{~g} / 100 \mathrm{~g}, 0.78$ and 2.06 , respectively. The results achieved suggest that 
27 the vis-NIR spectroscopy is a powerful technique for the quantification of glucose 28 adulteration in Saudi honey.

\section{Keywords}

Honey, adulteration, visible and near infrared spectroscopy, glucose, Saudi Arabia

INTRODUCTION

Honey has a wide range of applications in the food industry, food preservation or be used as an ingredient in hundreds of manufactured foods for its sweetness, colour, flavour, caramelisation and viscosity. ${ }^{1}$ Honey is an excellent source of energy, containing approximately $80 \mathrm{~g} / 100 \mathrm{~g}$ carbohydrates $(35 \mathrm{~g} / 100 \mathrm{~g}$ glucose, $40 \mathrm{~g} / 100 \mathrm{~g}$ fructose, and $5 \%$ sucrose) and $20 \mathrm{~g} / 100 \mathrm{~g}$ water. Also, it contains more than 180 substances, including amino acids, vitamins, minerals, enzymes, organic acids and phenol compounds. ${ }^{2}$ It is also consumed fresh in large amounts due to the associated health and medical characteristics. The high sugar concentration, low $\mathrm{pH}$ and the presence of flavonoids, hydrogen peroxide, phenolics and terpenes make honey a powerful antiseptic and antimicrobial agent useful in the treatment of burns, wounds, gastroenteritis stomach and skin ulcers. ${ }^{3,4}$ Due to its superior nutritional and health value and unique flavour, natural bee honey is preferred by consumers hence the price of bee honey is much greater than other sweetening commodities, such as refined cane sugar and corn syrup. The high price of the natural honey encourages workers in the honey industry, including beekeepers and merchants to adulterate honey worldwide, which leads to deterioration of honey quality, but increase honey quantity that is sold at the same price of natural authentic honey. Adulteration of bee honey with cheaper sweetening materials has been widely reported in 53 the literature.$^{5-7}$ In Saudi Arabia, honey adulteration is performed by mixing with cheap 54 imported honey, diluting with water, and/or addition of glucose syrup. Sometimes producers 
of authentic honey are obliged to artificially feed bees with glucose syrups, due to the lack of natural flora or the cost associated with moving the bee colony to areas rich with natural flora. Since the price of the Saudi natural honey is a multiple of ten for that of adulterated honey, a robust detection method of honey adulteration with glucose syrup will have a clear economic impact on Saudi consumers.

To guarantee authenticity of honey and protect the consumer from commercial exploitation, the quality of honey must be controlled analytically. ${ }^{8}$ Strict standards for commercial honey were set in various countries, based on specific physical properties and chemical compositions acquired with traditional analytical methods. However, Saudi Arabia currently lacks such standards, which emphasizes the importance of this research to Saudi Arabian honey market. Since analytical methods require extensive sample preparation and experienced operators, they are time consuming, expensive and destructive methods.

Spectroscopic techniques are advantageous over traditional methods, including mid infrared (MIR), fluorescence and visible and near infrared (vis-NIR) spectroscopy. Although vis-NIR has been used intensively for sugar analysis in food sector (e.g. He et al. ${ }^{9}$ ), less publication about the use of this technique in honey can be found in the open literature. Ruoff et al. ${ }^{10}$ reported accurate measurement of fructose, glucose, sucrose and maltose as well as the fructose/glucose and glucose/water ratios in honey samples from different crops. ${ }^{10,11}$ Quiu et al. ${ }^{13}$ determined the main chemical composition of commercial honey such as moisture, fructose, glucose, sucrose, and maltose with $R^{2}$ values of $1.0,0.97,0.91,0.86$, and 0.93 , respectively. Kelly et al. ${ }^{14}$ implemented the NIR coupled with principal component analysis to detect adulteration of Irish honey by beet invert syrup and high fructose corn syrup. Adulterated honey samples were well discriminated from authentic samples, particularly at a high fructose level of $10 \mathrm{~g} / 100 \mathrm{~g} \mathrm{w} / \mathrm{w}$. The NIR was also used to detect honey adulteration by addition of fructose and glucose. ${ }^{15}$ Similarly, adulteration of Mexican honey by sugar syrups such as corn syrup and cane sugar syrup was successfully detected with the NIR spectroscopy. ${ }^{16}$ This brief literature review demonstrates that no report is available on the use of the vis-NIR spectroscopy for the detection and quantification of glucose syrup 
adulterated in Saudi honey, although this is needed in a country that lacks standards for quality control of commercial honey.

The aim of this paper was to quantify adulteration with glucose syrup in authentic Saudi honey and imported honey in the Saudi market.

\section{MATERIALS AND METHODS}

\section{Honey Samples}

A total of 69 honey samples were collected and stored at a room temperature. The majority of these samples (56 samples) were produced in the Kingdom of Saudi Arabia. Among these samples, 32 authentic samples, produced in different regions of Saudi Arabia (Fig. 1) were collected directly from the beekeepers with guaranteed quality. The majority of these samples were from the southern part of the kingdom. Other 13 authentic Saudi samples were produced with bees fed complementary with glucose syrup. The intention of the beekeepers was not to adulterate honey to increase the profit, but to feed bees when natural feed lacks in the fields. These samples were collected from the northern and southern part of the Kingdom. The remaining 11 Saudi samples were commercially available in the Saudi market without guaranteed quality. The majority of these samples were from the western part of the country, except two samples from the central and southern parts. The remaining 13 samples were non-Saudi samples, which were imported from different countries into Saudi Arabia.. It is worth noting that none of these imported samples (except one sample from Egypt) is from any neighbouring countries of the Kingdom, indicating different floras than that of the Saudi Arabia..

Honey Adulteration with Glucose 
110 In order to evaluate the potential of the vis-NIR spectroscopy to quantify honey adulteration

111 with glucose, glucose syrup of four different concentrations of $5,12,19$ and $33 \mathrm{~g} / 100 \mathrm{~g}$ were 112 added to the honey samples. This practice is common in Saudi Arabia and in other countries 113 in the Middle East and North Africa. The sugar solution was prepared by weight as $1: 1$ of 114 glucose:water solution. After adding the sugar to water, the mixture was stirred properly at the boiling temperature, until the sugar melted completely in the solution. The solution was left to cool down before it was added to the honey samples. The honey samples were

117 liquefied in a heating cabinet at $30^{\circ} \mathrm{C}$ for $2-5$ hours to allow for cooling down before optical scanning with the vis-NIR spectrophotometer took place.

Visible and Near Infrared Spectroscopy and Scanning of Honey Samples

Optical scanning was carried out with AgroSpec mobile, fibre type, vis-NIR spectrophotometer (tec5 Technology for Spectroscopy, Oberursel, Germany), with a measurement range of $305-2200 \mathrm{~nm}$. The measurement in trans-reflectance mode was chosen in this study to measure honey spectra, as this measurement mode was recommended by other researchers. ${ }^{15}$ The data logging system consisted of tec5 analogue to digital converter data acquisition hardware and AgroSpec software (tec5 AG, Oberursel,

128 Germany). The light source was a separate 20 watt halogen lamp that illuminated the honey samples by means of optical fibres. An A40 reflection probe from tec5 (tec5 AG, Oberursel, Germany) was used to illuminate and collect the trans-reflected light from honey samples. Before scanning, several experimental trials were attempted until an optimised measurement set up was achieved (Fig. 2). The trans-reflectance measurement was performed using a ceramic white plat, on which a honey sample $(1-2 \mathrm{~mm}$ thick) was placed. The light penetrates the honey sample and reaches the white plate, from which light reflects through the honey sample back to the optical probe. A $100 \%$ ceramic was used as the white 
reference, which was scanned once every 30 minutes. From each honey sample three replicates were prepared, of each 10 scans were collected, and averaged in one spectrum.

Principal Component Analysis

The principal component analysis (PCA) was applied on the vis-NIR spectra recorded on selected honey samples to discriminate between non-adulterated and adulterated honey with glucose syrup with different ratio of concentrations of 5, 12, 19 and $33 \mathrm{~g} / 100 \mathrm{~g}$. In order to allow more detailed analysis with PCA, only 75 honey spectra of 15 randomly selected honey samples and their four adulterated versions were selected. PCA transforms the original independent variables (wavelengths) into new axes, or principal components (PCs). These PCs are orthogonal, so that the datasets presented on these axes are uncorrelated with each other. ${ }^{17,18}$ Therefore, the PCA expresses the total variation in the dataset in only a few PCs and each successively derived PC expresses decreasing amounts of the variance. The first PC covers as much of the variation in the data as possible. The second PC is orthogonal to the first PC and covers as much of the remaining variation as possible, and so on. By plotting the PCs, one can view interrelationships between different variables, and detect and interpret sample patterns, groupings, similarities, or differences. Similarity maps allow comparison of the spectra in such a way that two neighbouring points represent two similar spectra. It was found that maximum normalization was the best pre-treatment to provide a detailed discretion of the spectra variation, and this resulted in the best performance of PCA. Spectral pre-treatments and PCA were carried out using Unscrambler 7.8 software (Camo Inc., Oslo, Norway).

\section{Partial Least Squares Regression Analysis}

Before conducing partial least squares (PLS) regression analysis, pre-treatment of honey spectra was carried out. The spectral pre-treatment aimed at removing the noisy part in the 
spectrum or eliminating some sources of variation not related to the measured value. Different spectra pre-treatments were tested, and the pre-treatment that resulted in the best result was withheld. The first step in spectra pre-treatment was noise cut at both edges of honey spectra, which resulted in a spectral range of 340 to $2148 \mathrm{~nm}$. More data points were removed from the high end of the spectra because of the low signal-to-noise ratio at that end. - Noise cut was successively followed by (a) spectra wavelength reduction by averaging 4 adjacent wavelengths, which resulted in 453 wavelength variables, (b) baseline correction with baseline offset method, (c) $1^{\text {st }}$ derivation with the Savitzky-Golay algorithm based on the second-order polynomial, and (d) smoothing with the Savitzky-Golay method. The PLS regression analysis was used to develop quantitative models to predict the amount of artificially added glucose content in adulterated honey samples. The PLS is a bilinear modelling method where information in the original $x$ data is projected onto a small number of underlying ("latent") variables called PLS components. The y data are actively used in estimating the "latent" variables to ensure that the first components are the most relevant for predicting the $y$ variables. Interpretation of the relationship between $\mathrm{x}$ data and $\mathrm{y}$ data is then simplified as this relationship is concentrated on the smallest possible number of components. More detailed information about the PLS can be found in Martens and Naes. ${ }^{18}$

A total of 345 spectra of 69 non-adulterated and 276 adulterated honey samples with five glucose concentrations $(0,5,12,19$ and $33 \mathrm{~g} / 100 \mathrm{~g})$ were randomly divided into calibration (70 \%) and prediction (30\%) sets. The first group was used for the establishment of the PLS model, whereas the second group was used for model validation. The leave-one-out crossregression analysis. 
192 For the evaluation of the model performance, root mean square error of prediction (RMSEP)

193 in calibration and validation was used. ${ }^{19}$ The RMSEP can be expressed as follows:

$195 \quad \operatorname{RMSEP}=\sqrt{\frac{1}{\mathrm{~N}} \sum_{\mathrm{i}=1}^{\mathrm{N}}\left(\mathrm{X}_{\mathrm{i}}-\mathrm{Y}_{\mathrm{i}}\right)^{2}}$

196

197 where $X_{i}$ is the predicted value and $Y_{i}$ is the observed value.

198 Ratio of prediction deviation (RPD), which is the ratio of standard deviation (SD) of the measured values to RMSEP was used to compare between different calibration models developed. The third parameter considered was the coefficients of determination $\left(R^{2}\right)$. In fact, $R^{2}$ indicates the percentage of the variance in the $Y$ variable that is accounted for by the $\mathrm{X}$ variable. A value for $R^{2}$ between 0.50 and 0.65 indicates that more than $50 \%$ of the variance in $\mathrm{Y}$ is accounted for by variance $\mathrm{X}$, so that discrimination between high and low concentrations can be made. In the successful analysis of agricultural commodities, it is desirable to have $R^{2}>0.50$ and RPD $>5$. Nevertheless, for samples of complex material, Williams and Norris $^{19}$ classified values as follows: RPD $<1.0$ indicates very poor model/predictions and their use is not recommended; RPD between 2.4 and 3.0 indicates poor model/predictions where only high and low values are distinguishable; RPD between 3.1 and 4.9 indicates fair model/predictions, which may be used for assessment and correlation; RPD values between 5.0 and 6.4 indicates good model/predictions where quantitative predictions are possible; RPD between 6.5 and 8.0 indicates very good, quantitative model/predictions, and RPD > 8.1 indicates excellent model/predictions. Others reported that RPD is desired to be larger than 2 for a good calibration. ${ }^{20,21}$ These reports considered RPD ratio $\leq 1.5$ to indicate incorrect model predictions to prevent further prediction. Due to the conflicting information available in the literature about RPD values and the absence of information about RPD limits in honey, it was proposed to consider the 217 following RPD values: 
- $\quad$ RPD $\leq 1.5:$ Poor model accuracy.

- $\quad \mathrm{RPD}=1.5-2$ : moderate model prediction accuracy, where discrimination between high and low values can be made.

- $\quad$ RPD $=2$ - 2.5: good model prediction

- $\quad$ RPD $=2.5-3:$ very good model prediction, and

- $\quad$ RPD $\geq 3$ : Excellent model prediction.

The RPD values obtained in this study was classified according to the above proposed limits, and were used to evaluate the accuracy of PLS models for the prediction of glucose content in adulterated honey samples.

\section{RESULTS AND DISCUSSION}

\section{Characteristics of Honey Spectra}

Figure (3) shows an average spectrum of the authentic honey samples (32 samples). The spectrum illustrates a typical spectral feature and absorption bands to those reported for honey. ${ }^{22}$ Several absorption peaks (dips on Fig. 3) of different amplitude can be distinguished. A clear absorption peak in the vis range at $400 \mathrm{~nm}$ is observed, which can be attributed to colour variation of honey samples. Two absorption peaks appearing at 984 and $1450 \mathrm{~nm}$ in the NIR range are attributed to the $\mathrm{O}-\mathrm{H}$ absorption bands at the third and second overtones, respectively. However, the absorption peak at $1450 \mathrm{~nm}$ is much larger and obvious. A third and largest $\mathrm{O}-\mathrm{H}$ absorption band at 1930 is attributed to the $\mathrm{OH}$ stretch + $\mathrm{OH}$ bending. The fourth absorption peak in the NIR region locates at about $1170 \mathrm{~nm}$ and corresponds to $\mathrm{C}-\mathrm{H}$ stretching in the second overtone region. This waveband is similar to the corresponding waveband observed by Shenk et al. ${ }^{23}$ at 1150 . Two small dips at 1688 and $1760 \mathrm{~nm}$ might be attributed, respectively, to $\mathrm{CH} 2$ anti-symmetric stretching and $\mathrm{CH} 3$ symmetric stretching in the first overtone region. Shenk et al. ${ }^{23}$ reported that $1700 \mathrm{~nm}$ waveband is associated with $\mathrm{C}-\mathrm{H} 1^{\text {st }}$ overtone, which is comparable to that at $1688 \mathrm{~nm}$ 
246 observed in this study. Murray and Williams ${ }^{24}$ attributed the spectral features around 1720

$247 \mathrm{~nm}$ to $\mathrm{C}-\mathrm{H}$ bond of carbonyl compounds. The absorption band at $2102 \mathrm{~nm}$ is assigned to C-

$248 \mathrm{H}$ deformation and combination or $\mathrm{C}-\mathrm{O}$ stretch combination overtones and was assigned to

249 carbohydrate in honey. ${ }^{25-27}$ Protein has also a reflectance bands peak at about $2180 \mathrm{~nm}$,

250 which was not detectable in the current study. ${ }^{21}$

251 Figure (4) compares average spectrum of non-adulterated honey with four average spectra

252 of adulterated honey samples with four sugar concentrations of $5,12,19$ and $33 \mathrm{~g} / 100 \mathrm{~g}$.

253 Clear differences can be observed between spectra of adulterated honey samples, as

254 compared to the non-adulterated sample. The authentic non-adulterated spectrum

255 demonstrates less reflectance and higher absorption, as compared to glucose-adulterated

256 spectra. Generally, in the visible range, reflectance increases with the amount of glucose

257 added to the adulterated samples, which might be attributed to changes in honey colour.

258 With increasing the amount of glucose syrup added, honey samples become lighter in

259 colour, hence, reflectance increases due decreasing the overall absorption. However, in the

260 NIR range mixed behaviour is observed, which is difficult to be explained. The water

261 absorption band particularly at $1950 \mathrm{~nm}$ is more evident in the adulterated honey spectra, as

262 compared to the non-adulterated spectra.

263

Discrimination between Adulterated from Non-Adulterated Honey Samples

Figure (5a) shows PC similarity map (PCs 1 and 2), resulted from PCA carried out on normalised honey spectra of adulterated and non-adulterated samples. A good separation is observed between the 15 non-adulterated samples from the adulterated samples. One observation may be made about this perfect separation is the diagonal direction of separation, with non-adulterated samples locate on the left and bottom sides of PC2 and PC1, respectively. Separation along PC1 appears to be more pronounced, as compared to separation along PC2. However, minor overlap can be observed. Since adulteration with 5 $\mathrm{g} / 100 \mathrm{~g}$ glucose syrup is considered as small concentration, ${ }^{15}$ it might be difficult to be 
274 detected by the vis-NIR spectroscopy. Therefore, by excluding samples with $5 \mathrm{~g} / 100 \mathrm{~g}$ 275 adulteration rate, from the PCA enables perfect separation (Fig. 5b). This result proves the 276 capability of vis-NIR spectroscopy coupled with PCA to discriminate between authentic honey samples from the corresponding adulterated samples with different glucose contents of 12,19 and $33 \mathrm{~g} / 100 \mathrm{~g}$. However, samples of different ratios of glucose concentrations are completely overlapped, which is not a good sign for the sensitivity of the vis-NIR spectroscopy to quantify the amount of sugar used to adulterate honey.

Glucose Quantification in Adulterated Honey Samples

Table 2 summarises results of PLS regression analysis for the prediction of glucose content in adulterated honey samples in the cross-validation and in the prediction sets. Values of RPD of 2.56 and 2.06 obtained, respectively, for the calibration and the prediction sets indicate very good and good model predictions, respectively. This result is in-line with results reported in the literature for the prediction of glucose ratio used to adulterate honey samples. Ruoff et. al. ${ }^{9}$ obtained $R^{2}$ values in the range of $0.81-0.88$, which is in the same range of the current study $(0.78-0.85)$ for both the cross-validation and prediction sets (Table 2). The scatter plot of measured versus predicted glucose concentrations in adulterated honey samples in the prediction set is shown in Fig. (6).

During the PLS regression analysis, the observed response values are approximated by a linear combination of the values of the predictors. The coefficients of that combination are called regression coefficients or B-coefficients. The regression coefficients plot is a useful plot to identify the important wavelengths for the prediction of a property. The absolute value of the regression coefficients is the largest for the wavelengths that contribute most to the prediction equation. This plot over the entire wavelength range shows distinguished wavelength bands for glucose prediction (Fig. 7). There are distinct absorption peaks throughout the vis and NIR regions, with the most significant are at two spectral ranges of $400-600$ (vis range) and $1900-2145 \mathrm{~nm}$ (NIR range). In the vis range significant 
wavebands at 428,532 and $580 \mathrm{~nm}$ are thought to be associated with colour changes. The peaks around 1700, 1150, 930 and $780 \mathrm{~nm}$ in the NIR range correspond to $\mathrm{C}-\mathrm{H}$ 1st, 2nd, 3rd and 4th overtones, respectively. ${ }^{23}$ Comparable bands in the NIR range to those found by Shenk et al. ${ }^{23}$ were observed in this study, respectively at 1756, 1160, 976 and $840 \mathrm{~nm}$. Other bands at 1000, 1456 and $1944 \mathrm{~nm}$ associate with O-H stretch in the third, second and first overtones, respectively, can also be observed. The large positive peak at $2020 \mathrm{~nm}$ and negative peak at $2076 \mathrm{~nm}$ can be assigned to carbohydrate. The absorption bands at 2102 $\mathrm{nm}$ is assigned to $\mathrm{C}-\mathrm{H}$ deformation and combination or $\mathrm{C}-\mathrm{O}$ stretch combination overtones and were both assigned to carbohydrate in honey. ${ }^{25-27}$ These detailed information about significant wavebands for the detection of glucose adulteration derived from the correlation coefficient plots are in agreement with those for the honey raw spectra reported in this paper and in the literature.

\section{CONCLUSIONS}

This study evaluated the potential of the visible and near infrared (vis-NIR) spectroscopy coupled with chemometrics for the detection of glucose adulteration in Saudi honey. Honey spectral features enabled clear discrimination between non-adulterated and adulterated honey samples. Larger overall absorption and smaller water absorption bands characterised the non-adulterated spectra, as compared to the adulterated samples. The vis-NIR spectroscopy coupled with principal component analysis (PCA) was found to be a useful technique to discriminate between non-adulterated from adulterated samples with glucose ratios of 12,19 and $33 \mathrm{~g} / 100 \mathrm{~g}$. However, small overlap was observed between the two sample groups only at small concentration of glucose syrup of $5 \mathrm{~g} / 100 \mathrm{~g}$. The vis-NIR spectroscopy coupled with partial least squares (PLS) regression enabled the prediction of the amount of glucose added to the adulterated honey samples with very good to good model prediction accuracy. The regression coefficients plot obtained from PLS regression showed distinguished bands in the visible range, associated with colour changes and in the 
$330 \mathrm{NIR}$ range, associated with $\mathrm{C}-\mathrm{H}, \mathrm{C}-\mathrm{O}, \mathrm{O}-\mathrm{H}$ bonds; both reflected the addition of glucose

331 syrup to the honey samples. More advanced mathematical modelling techniques, e.g. 332 artificial neural network and support vector machine that can handle the non-linear responses in the dataset are recommended for future work to improve the prediction accuracy of glucose concentration in adulterated Saudi honey samples.

\section{ACKNOWLEDGMENT}

Authors acknowledge the financial supports received from the government of Kingdom of

Saudi Arabia.

\section{REFERENCES}

1. Rodriguez, G.O.; Ferrer, B.S.; Ferrer, A.; Rodriguez, B. Characterization of Honey Produced in Venezuela. Food Chemistry, 2004, 84, 499-502.

2. Ouchemoukh, S.; Louaileche, H.; Schweizer, P. Physicochemical Characteristics and Pollen Spectrum of Some Algerian Honeys. Food Control, 2007, 18, 52-58

3. McCarthy, J. The Antibacterial Effects of Honey: Medical Fact or Fiction? American Bee Journal, 1995, 135, 341-342.

4. Dunford, C.; Cooper, R.; Molan, P.; White, R. The Use of Honey in Wound Management. Nurs Standard, 2000, 15(11), 63-8.

5. Cienfuegos, E.; Casar, I.; Morales, P. Carbon Isotopic Composition of Mexican Honey. Journal of Apiculture Research, 1997, 36, 169-179.

6. Tien, L.L.; Shau, M.O.A. Quality Analysis of Longan Honey in Taiwan Market. Food Science Taiwan, 1997, 24, 479-489.

7. Gonzalez, M.I.; Marques, M.E.; Sanchez, S.J.; Gonzalez, R.B. Detection of Honey Adulteration with Beet Sugar Using Stable Isotope Methodology. Food Chemistry, 1997, 61(3), 281-286. 
8. Mendes, E.; Brojo Proenca, E.; Ferreira, I.M.P.L.V.O.; Ferreira, M.A. Quality Evaluation of Portuguese Honey. Carbohydrate Polymers, 1998, 37, 219-22.

9. He, Y.; Wu, D.; Feng, S.; Li, X. Fast Measurement of Sugar Content of Yogurt Using Vis/NIR-Spectroscopy. International Journal of Food Properties, 2007, 10(1), 1-7.

10. Ruoff, K.; Luginbuehl, W.; Bogdanov, S.; Bosset, J.O.; Estermann, B.; Ziolko, T.; Kheradmandan, S.; Amado, R. Quantitative determination of physical and chemical measurands in honey by near-infrared spectrometry. European Food Research and Technology, 2007, 225, 415-423

11. Cho, H.J.; Hong, S.H. Acacia Honey Quality Measurement by Near Infrared Spectroscopy. Journal of Near Infrared Spectroscopy, 1998, 6, A329-A331.

12. Garcia Alvarez, M.; Ceresuela, S.; Huidobro, J.F.; Hermida, M.; Rodriguez Otero, J.L. Determination of Polarimetric Parameters of Honey by Near-Infrared Transflectance Spectroscopy. Journal of Agriculture and Food Chemistry, 2002, 50, 419-425P.

13. Quiu, P.Y.; Ding, H.B.; Tang, Y.K.; Xu, R.J. Determination of Chemical Composition of Commercial Honey by Near-Infrared Spectroscopy, Journal of Agriculture and Food Chemistry, 1999, 47, 2760-2765.

14. Kelly, J.D.; Downey, G.; Fouratier, V. Potential of Near Infrared Spectroscopy to Detect Adulteration of Irish Honey Samples by Beet Inverted Syrup and High Fructose Corn Syrup. Journal of Near Infrared Spectroscopy, 2006, 14(2): 139-146.

15. Downey, G.; Fouratier V.; Kelly J.D. Detection of Honey Adulteration of Fructose and Glucose Using Near Infrared Transflectance Spectroscopy, Journal of Near Infrared Spectroscopy, 2003, 11, 447-456.

16. Rios-Corripio, M.A.; Rios-Leal, E.; Rojas-López, M.; Delgado-Macuil, R. FTIR Characterization of Mexican Honey and its Adulteration with Sugar Syrups by Using Chemometric Methods. Journal of Physics: Conference Series, 2011, 274(1), 012098 I.

17. Jolliffe, I.T. Principal Component Analysis. Springer, New York, 1986; 393 pp.

18. Martens, H.; Naes, T. Multivariate Calibration. 2nd ed. John Wiley \& Sons, Chichester, UK, 1989; 419 pp. 
19. Williams, P.C.; Norris, K. Variables Affecting Near-infrared Spectroscopic Analysis. In Near-Infrared Technology in Agricultural and Food Industries; Williams, P.C.; Norris, K. eds.; American Association of Cereal Chemists, Inc. St. Paul Minnesota, USA, 2001, 171-189.

20. Williams, P. Near-Infrared Technology—Getting the Best Out of Light. A Short Course in the Practical Implementation of Near Infrared Spectroscopy for User. PDK Grain Project, Inc., Nanaimo, Canada, 2008.

21. Karoui, R.; Mouazen, A.M.; Dufour, É.; Pillonel, L.; Schaller, E.; De Baerdemaeker, J.; Bosset, J-O. Chemical determination of European Emmental cheeses by near infrared spectroscopy using chemometric tools. International Dairy Journal, 2006, 16(10), 1211 1217.

22. Webster, T.C.; Dowell, F.E.; Maghirang, E.B.; Thacker, E.M. Visible and Near-Infrared Spectroscopy Detects Queen Honey Bee Insemination. Apidologie, 2009, 40(5), 565569.

23. Shenk J.S.; Workman J.J.; Westerhaus M.O., 2001. Application of NIR Spectroscopy to Agricultural Products. In Handbook of Near-Infrared Analysis; Burns, D.; Ciurczak E. eds.; Marcel Dekker Inc, New York, NY, 2001, 419-474.

24. Murray, I.; Williams, P.C. Chemical Principles of Near Infrared Technology. In Near Infrared Technologies in Agricultural and Food industries; Williams, P.; Norris, K. eds., American Association of Cereal Chemists, St. Paul, MN, The USA, 2001, 17-31.

25. Osborne, B.G.; Fearn, T.; Hindle, P.H. Practical NIR Spectroscopy with Application in Food and Beverage Analysis. $2^{\text {nd }}$ Edn. Longman Scientific and Technical, Harlow, Essex, UK, 1993; 228 pp.

26. Cozzolino, D.; Smyth, H.E.; Gishen, M. Feasibility Study on the Use of Visible and Near Infrared Spectroscopy Together with Chemometrics to Discriminate between Commercial White Wines of Different Varietal Origins. Journal of Agriculture and Food Chemistry, 2003, 51, 7703-7708. 
27. Murray, I. The NIR Spectra of Homologous Series of Organic Compounds. In Proceedings International NIR/NIT Conference; Hollo, J.; Kaffka, K.J.; Gonczy, J.L. eds., Akademiai Kiado, Budapest, Hungary, 1986, 13-28.

(1)

(1)

6

7

8

9




\section{Table 1}

Sample statistics of glucose in the calibration and validation data sets

\begin{tabular}{lccccc}
\hline Data set & Number of & Minimum, & Maximum, & Mean, & SD \\
& samples & $\mathrm{g} / 100 \mathrm{~g}$ & $\mathrm{~g} / 100 \mathrm{~g}$ & $\mathrm{~g} / 100 \mathrm{~g}$ & \\
\hline Calibration data set & 242 & 0 & 33 & 13.75 & 11.48 \\
Validation data set & 103 & 0 & 33 & 13.75 & 11.48 \\
\hline
\end{tabular}

SD is the standard deviation 

Abdul M. Mouazen; Nourah M. Alwaalan

Table 2.

512 Results of cross-validation and independent validation for the prediction of glucose concentration in adulterated honey samples

\begin{tabular}{|c|c|c|c|c|c|}
\hline Validation & $R^{2}$ & Slope & SD & $\begin{array}{l}\text { RMSEP, } \\
\mathrm{g} / 100 \mathrm{~g}\end{array}$ & RPD \\
\hline Cross-validation & 0.85 & 0.86 & 11.47 & 4.52 & 2.54 \\
\hline Independent validation & 0.78 & 0.85 & 11.48 & 5.56 & 2.06 \\
\hline
\end{tabular}

RMSEP is root mean squares error of prediction, 